University of Nebraska - Lincoln

DigitalCommons@University of Nebraska - Lincoln

July 1998

\title{
Structure and lattice dynamics of dipolarly disordered 2,3-dimethylanthracene crystals
}

\author{
M. Dörr \\ Experimentalphysik I, Universität Bayreuth, D-95440 Bayreuth, Germany \\ H. Gerlach \\ Organische Chemie II, Universität Bayreuth, D-95440 Bayreuth, Germany \\ J. Kalus \\ Experimentalphysik I, Universität Bayreuth, D-95440 Bayreuth, Germany
}

N. Karl

Physikalisches Institut, 3 Universität Stuttgart, Pfaffenwaldring 57, D-70569 Stuttgart, Germany

M. Monkenbusch

Forschungszentrum Jüulich GmbH, Institut für Festkörperforschung, D-52425 Jülich, Germany

See next page for additional authors

Follow this and additional works at: https://digitalcommons.unl.edu/chemistrystezowski

Part of the Chemistry Commons

Dörr, M.; Gerlach, H.; Kalus, J.; Karl, N.; Monkenbusch, M.; Natkaniec, I.; Schmelzer, U.; Schmidt, W.; Stezowski, John J.; Vorderwisch, P.; and Warth, M., "Structure and lattice dynamics of dipolarly disordered 2,3-dimethylanthracene crystals" (1998). John J. Stezowski Publications. 8.

https://digitalcommons.unl.edu/chemistrystezowski/8

This Article is brought to you for free and open access by the Published Research - Department of Chemistry at DigitalCommons@University of Nebraska - Lincoln. It has been accepted for inclusion in John J. Stezowski Publications by an authorized administrator of DigitalCommons@University of Nebraska - Lincoln. 
Authors

M. Dörr, H. Gerlach, J. Kalus, N. Karl, M. Monkenbusch, I. Natkaniec, U. Schmelzer, W. Schmidt, John J. Stezowski, P. Vorderwisch, and M. Warth 


\title{
Structure and lattice dynamics of dipolarly disordered 2,3-dimethylanthracene crystals
}

\author{
M. Dörr ${ }^{1}$, H. Gerlach², J. Kalus ${ }^{1}$, N. Karl ${ }^{3}$, M. Monkenbusch ${ }^{4}$, \\ I. Natkaniec ${ }^{5}$, U. Schmelzer ${ }^{1}$, W. Schmidt ${ }^{6}$, J. J. Stezowski ${ }^{7}$, \\ P. Vorderwisch ${ }^{8}$, G. Voss ${ }^{2}$, and M. Warth ${ }^{3}$ \\ ${ }^{1}$ Experimentalphysik I, Universität Bayreuth, D-95440 Bayreuth, Germany \\ ${ }^{2}$ Organische Chemie II, Universität Bayreuth, D-95440 Bayreuth, Germany \\ 3 Physikalisches Institut, 3 Universität Stuttgart, Pfaffenwaldring 57, D-70569 Stuttgart, \\ Germany \\ 4 Forschungszentrum Jüulich GmbH, Institut für Festkörperforschung, D-52425 Jülich, \\ Germany \\ 5 Joint Institute for Nuclear Research, PO Box 79, Dubna, 101000 Moscow, Russian \\ Federation \\ ${ }^{6}$ Institut Laue-Langevin, F-38042 Grenoble Cédex 9, France \\ ${ }^{7}$ Department of Chemistry, University of Nebraska-Lincoln, Lincoln, NE 68588-0304, USA \\ ${ }^{8}$ Hahn-Meitner-Institut Berlin, Berlin Neutron Scattering Centre, D-14109 Berlin, Germany
}

\begin{abstract}
DMA, $\left.\mathrm{C}_{16} \mathrm{H}_{14}\right)$ crystallizes in a pseudocentrosymmetric triclinic lattice with two molecules per unit cell showing dipolar structural disorder of the acentric molecules. Using powder samples the amplitude weighted phonon density of states was measured by inelastic incoherent neutron scattering for different degrees of deuteration. The differences in the resulting spectra can be explained by changes of the dynamics of the molecular bodies or the methyl side groups. By the method of inelastic coherent neutron scattering phonon dispersion curves were determined. The phonon lines are broad even at low temperatures; this fact is supposed to be related to dipolar disorder. Both the incoherent and coherent scattering results can be described satisfactorily by lattice dynamical calculations using a " 6 -exp" potential for the atom-atom interactions of different molecules, including internal degrees of freedom.
\end{abstract}

\section{Introduction}

The acentric 2,3-dimethylanthracene molecule, as will be shown by a full $\mathrm{x}$-ray crystal structure analysis, crystallizes in a pseudo-centrosymmetric triclinic lattice with two molecules in the unit cell. The non-centrosymmetric molecules are statistically disordered in such a way that the x-rays "see" a centrosymmetric molecule on average in the coherently scattering volume. This kind of disorder has been named "dipolar disorder" in a similar case, that of 2,3-dimethylnaphthalene [1]. It is unique in that only one degree of freedom, the direction of the polar long molecular axis, is disordered (up and down), while otherwise a well defined translationally periodic crystal lattice is formed. Having in mind the aim of studying lattice dynamics in disordered molecular crystals, this kind of well defined disorder appeared especially attractive. Though dipolarly disordered, the crystals are crystallographically perfect, giving rise to sharp Bragg reflections, and therefore are in many respects close to a perfect crystal, but some features which are typical of amor- 
phous structures are seen too: the widths of the phonon lines become broad. This is due to the fact that in the strict sense the translational invariance of the crystal is lost. Without translational invariance, force constants show a variance, and even with a harmonic force field, where the lifetime of a phonon becomes infinite, the measured width of a phonon becomes finite. Another consequence is the appearance of diffuse elastically scattered intensity between the Bragg reflections. The elastic intensity distribution measured via $\mathrm{x}$-ray scattering indeed can be explained on the basis of dipolar disorder. Recent investigations on likewise dipolarly disordered 2,3-dimethylnaphthalene have shown [2] that a remarkable correlation in orientation exists between next neighbours in the crystallographic $b$-direction. With a probability of 0.615 on average a molecule at position $[0,1$, $0]$ is oriented antiparallel with respect to the orientation of a molecule at position $[0,0,0]$. Orientational correlations to other neighbouring molecules also exist, but these are much less pronounced. Nevertheless, as a consequence of the experimentally found space group symmetry $P 2_{1} / a[1]$ the net orientational distribution of the molecules in the crystal has to be $50 \%$ up and $50 \%$ down.

We present here in detail the results of incoherent and coherent inelastic neutron scattering experiments which both give information about the lattice dynamics of a dipolarly disordered crystal. Some aspects of this work were presented as a conference report [3]. The measurement of incoherent inelastic neutron scattering spectra of 2,3-DMA with different degrees of deuteration yielded information about the phonon density of states $g(\omega)$. For example at $10 \mathrm{~K}$ a librational mode of the $\mathrm{CH} 3$ groups was identified at an energy of $5.5 \mathrm{THz}$. Its energy decreases to $4.2 \mathrm{THz}$ for deuterated methyl groups. A decrease of this amount is expected due to the change of the moment of inertia, provided the libration takes place around the axis of symmetry of the methyl group.

The measurement of coherent inelastic neutron scattering with a fully deuterated single crystal of 2,3-DMA gives information about the phonon dispersion curves. Probably for reasons of the dipolar disorder, which gives rise to the mentioned broadening of the phonon lines, we could only observe phonons with energies below $\sim 1.1 \mathrm{THz}$ up to now. We found, for example, that the energies of the acoustic phonon branches are softened by a remarkable amount compared to the acoustic branches of the parent hydrocarbon anthracene [4]. This result implies that the force constants used for an interpretation of the well understood lattice dynamics of anthracene cannot be transferred directly for an interpretation of the lattice dynamics of 2,3-DMA.

The paper is organized as follows: In section 2 we discuss the preparation of samples, describe the experimental setup used for the x-ray crystal structure analysis as well as that used for coherent and incoherent neutron scattering, and present the evaluation and the results of the measurements. An overview about calculations and models follows in section 3. Calculated dispersion curves are presented, and the measured branches are assigned to the predominant character of the respective motion of the molecules in the unit cell. A discussion of how the gas phase vibrations are influenced by the molecular lattice and conclusions are given in the last section.

\section{Experimental setup and results}

\subsection{Sample preparation}

2,3-dimethylanthracene subsequently abbreviated as $\left(2,3-\right.$ DMA- $\left._{14}\right)$ was synthesized as follows: 1,4,4a,9a-tetrahydro-2,3-dimethyl-9,10-anthraquinone (mp $145-148{ }^{\circ} \mathrm{C}$, lit: 150 ${ }^{\circ} \mathrm{C}[5]$ ) was synthesized from 1,4-naphthoquinone (Fluka No 70372) and 2,3-dimethyl1,3butadiene (Fluka No 39720) according to a previous report [5]; dehydrogenation with 
oxygen in ethanol/KOH [5] gave 2,3-dimethyl-9,10-anthraquinone $\left(\mathrm{mp} 208-210{ }^{\circ} \mathrm{C}\right.$, lit: 209-210 ${ }^{\circ} \mathrm{C}$ [5]). 2,3-dimethyl-9,10-anthraquinone was reduced with zinc in $33 \% \mathrm{NH}_{3}$ in $\mathrm{H}_{2} \mathrm{O}$ [6] to form 9,10-dihydro-2,3-dimethylanthracene (mp $124-126{ }^{\circ} \mathrm{C}$, lit: $122{ }^{\circ} \mathrm{C}$ [7]) which was dehydrogenated with $5 \% \mathrm{Pd} / \mathrm{C}$ [8] to yield the final product 2,3-DMA-h 14 (mp $250-252{ }^{\circ} \mathrm{C}$, lit: $\left.252{ }^{\circ} \mathrm{C}[9]\right)$.

1,4,5,6,7,8,9,10-octadeutero-2,3-dimethylanthracene $\left(2,3-\right.$ DMA-d $\left._{8}\right)$ : dimethyl-4,5-dimethyl-1,4-cyclohexadien-1,2-dicarboxylate ( $\mathrm{mp} 66-67^{\circ} \mathrm{C}$, lit: $\left.76^{\circ} \mathrm{C}[10]\right)$ was synthesized from dimethylacetylenedicarboxylate (Aldrich No 762-42-5) and 2,3-dimethyl-1,3butadiene [11]; dehydrogenation with $5 \% \mathrm{Pd} / \mathrm{C}\left(4.5 \mathrm{~h}, 200{ }^{\circ} \mathrm{C}\right)$ provided dimethyl-4,5dimethylbenzene-1,2-dicarboxylate ( $\mathrm{mp} 50-54{ }^{\circ} \mathrm{C}$, lit: $\left.56{ }^{\circ} \mathrm{C}[10]\right)$. Saponification to the dicarboxylic acid (mp 180-190 ${ }^{\circ} \mathrm{C}$, lit: $201{ }^{\circ} \mathrm{C}$ [11]) and subsequent reaction with acetic anhydride $\left(2 \mathrm{~h}, 165^{\circ} \mathrm{C}\right)$ formed 4,5-dimethyl-phthalic-anhydride (mp 212-213 ${ }^{\circ} \mathrm{C}$ [12]). 4,5-dimethylphthalic-anhydride was reacted with benzene- $\mathrm{d}_{6}$ [13] yielding $2-(2,3,4,5,6$ pentadeuterobenzoyl)-4,5-dimethyl-benzoic-acid (mp 180-190 ${ }^{\circ} \mathrm{C}$, lit: $197.5^{\circ} \mathrm{C}$ [14]); the latter was converted according to [15] with polyphosphoric acid into 2,3-dimethyl-5,6,7,8tetradeutero-9,10-anthraquinone ( $\mathrm{mp} 200-202{ }^{\circ} \mathrm{C}$, lit: $210.6-211.5{ }^{\circ} \mathrm{C}$ [16]). 2,3-dimethyl5,6,7,8tetradeutero-9,10-anthraquinone was then reduced to 9,10-dihydro-2,3-dimethyl5,6,7,8tetradeuteroanthracene $\left(\mathrm{mp} 122-128{ }^{\circ} \mathrm{C}\right.$, lit: $122{ }^{\circ} \mathrm{C}$ [7]) with zinc $/ 33 \% \mathrm{NH}_{3}$ in $\mathrm{H}_{2} \mathrm{O}$ [6]. 9,10-dihydro-2,3-dimethyl-5,6,7,8-tetradeuteroanthracene was reacted in a closed glass tube to hydrogen/deuterium exchange [17] under dehydrogenation with $65 \% \mathrm{D}_{2} \mathrm{SO}_{4}(10 \mathrm{~d}$, $130{ }^{\circ} \mathrm{C}$ ) to yield the desired 2,3-DMA- $\mathrm{d}_{8}$.

Perdeutero-2,3-dimethylanthracene (2,3-DMA-d14): this compound was prepared analogously to 2,3-DMA- $\mathrm{d}_{8}$, but with 2,3-dimethyl-1,3-butadiene- $\mathrm{d}_{10}$ and dimethylacetylenedicarboxylate as starting materials. The perdeuterated 2,3-dimethyl-1,3-butadiene was prepared from hexadeuteroacetone via hexadeuteropinacol [18] according to [19]. The degree of deuteration of C14D8(CD3)2, "2,3-DMA-d 14 ," was $93 \%$ as determined by ${ }^{1} \mathrm{H}$ NMR spectroscopy. The crude product was zone-refined according to standard techniques [20]; the colour changed from an initially dark melt via red to a pale yellow with increasing number of molten zone passages. The melting point of the purest fraction of zonerefined material amounted to $254^{\circ} \mathrm{C}$.

Single crystals could be grown from the melt in evacuated Pyrex glass ampoules by the Bridgman technique at a speed of $\sim 1 \mathrm{~mm} \mathrm{~h}^{-1}$ [20]. To avoid formation of decomposition byproducts it was necessary to keep temperatures close to the melting point. The Bridgman ingots obtained were composed of large (typically up to $15 \times 20 \mathrm{~mm}^{2}$ ) plate-shaped single crystalline domains of $1-3 \mathrm{~mm}$ thickness with slight mutual misorientations, but with the large plane always more or less parallel to the growth direction. Single crystalline plates with cleaved surfaces appeared polarization-optically perfect and showed a biaxial interference pattern with one bisectrix in the viewing field and distinct isochromatic lines up to high order, when viewed conoscopically (numerical aperture 0.85 ) in monochromatic light; twins were easily distinguishable from a superposition of interference patterns. For $\mathrm{x}$-ray crystal structure analysis samples of typically $0.5 \times 0.5 \times 0.5 \mathrm{~mm}^{3}$ where cut-off by a dissolution thread saw using a very thin cotton thread and xylene as a solvent.

\subsection{X-ray crystal structure analysis}

An initial set of lattice parameters was obtained from Buerger precession photographs. For a full crystal structure analysis a four circle diffractometer (Syntex $P 1$ ) was used in $\omega$-scan mode with monochromatized Mo K $\alpha$ radiation, $\lambda=0.071069 \mathrm{~nm}$. The unit cell was determined from refinement of 21 selected automatically centred x-ray reflections $\left(20^{\circ}<2 \Theta\right.$ $<30^{\circ}, T=296 \mathrm{~K}$ ), using the program packet The X-RAY SYSTEM, Version of 1976 [21], 
which yielded

$$
\begin{aligned}
& a=0.78400(8) \mathrm{nm} \quad \alpha=93.20(5) \quad V=0.56422 \mathrm{~nm}^{3} \\
& b=0.59796(6) \mathrm{nm} \quad \beta=105.62(3)^{\circ} \\
& c=1.25179(15) \mathrm{nm} \quad \gamma=89.997(9)
\end{aligned}
$$

The assumption of $Z=2$ molecules in the unit cell gave a reasonable density of $1.2 \mathrm{~g} \mathrm{~cm}^{-3}$.

In a subsequent fully automatized data collection 2391 reflections in the range $0<$ $2 \Theta<55^{\circ}$ were measured. Each reflection was scanned in an angular range of $0.75^{\circ}$ with a scanning speed adapted to the intensity. The background on both sides was registered with $50 \%$ of the scan time. For three reference reflections intensity was rechecked every 200 reflections. Eight reflections required measurement after attenuation by a copper foil because of too high intensity. Only 439 reflections obeyed the usual signal to noise criterion $I>3 \sigma(I)$; therefore a number of weaker reflections was included in the structure determination. The raw data were corrected for polarization and Lorentz factors. Absorption of the small, nearly isometric organic hydrocarbon crystal could be neglected.

From a Wilson plot centrosymmetry was concluded. Nevertheless, the refinement was started assuming $P 1$ symmetry. Only as soon as a centre of symmetry could be uniquely fixed during structure refinement was $P \overline{1}$ symmetry adopted (see below).

A Patterson analysis gave several intramolecular vectors at lengths as expected from the chemical structure formula. However, the vectors $0.07,0.21,0.12$ and $0.36 \mathrm{~nm}$ found in addition could not be explained with an ordered crystal structure. In analogy with the crystal structure of the next smaller homologue, 2,3-dimethylnaphthalene, determined earlier [1], dipolar disorder was therefore assumed as a most plausible cause here too, i.e. a statistical $50 \% / 50 \%$ distribution in space of parallel and antiparallel orientations of the long molecular axis (in conjunction with a parallel orientation of the molecular planes). For a statistical equipartition of these up and down molecules within the coherently scattering volume the x-rays "see" a pseudo-centre of symmetry. The additional vectors are indeed explainable by a superposition as sketched on the left side of figure 3 (see below).

Trials to further refine the crystal structure by difference Fourier synthesis were unsuccessful. The tight effective electron distribution brought about by superposition of the second molecular orientation and position precluded a resolution of the individual molecular geometry. Therefore the further evaluation was restricted to a rigid body refinement, using a molecular structure derived from the experimental one of an anthracene molecule to which the methyl carbon atoms were attached at a distance of $0.1510 \mathrm{~nm}$ from the appropriate ring $\mathrm{C}$ atoms 2 and 3 under exocyclic angles of $120^{\circ}$. Further refinement of the obtained atomic positions of $64 \mathrm{C}$ atoms in the bimolecular unit cell with superposition of two orientations in each molecular position was performed with the program CRYSLQ, still in $P 1$. The obtained coordinates located a centre of symmetry in the molecular plane(s). Three-dimensional refinement of the displacement of the second molecule in the statistically inversion symmetric pair led to a best shift value of $\Delta=0.12 \mathrm{~nm}$ (i.e. half a benzene ring) along the long molecular axis. The statistical superposition of two non-inversionsymmetric molecules on every site explains the additional vectors found in the Patterson analysis. The origin of the crystallographic coordinate system was therefore finally shifted to the inversion centre and $P \overline{1}$ symmetry was adopted.

In a final refinement the general isotropic temperature factor obtained from the Wilson plot was replaced by individual isotropic temperature factors for each carbon atom and then by anisotropic ones. In addition, the scaling factor was optimized. An $R$-value of $11.4 \%$ could be achieved, which increased to $13.5 \%$ when a weighted scheme (program WTLSSQ) was applied. The relatively high $R$-value was considered reasonable in view 
of the dipolar disorder in the crystal structure, the use of a rigid body molecular structure and the high percentage of low intensity reflections involved. Further, the hydrogen atoms had not been included in the refinement and a simultaneous refinement of the atomic coordinates, the temperature factors and the scaling factor had not been possible. The fractional atomic coordinates as well as the temperature factors at room temperature are presented in table 1 . The atomic numbering scheme and the molecular geometry used for the rigid body refinement are plotted in figure 1 . The two independent molecules A and B lie in the planes

$$
\begin{aligned}
0.885 x+0.446 y+0.138 z & =-0.001[\AA] \\
0.882 x-0.445 y+0.157 z & =-2.127[\AA]
\end{aligned}
$$

where $x, y, z$ are orthonormalized coordinates (in $\AA$ ) along $a, c \times a$ and $a \times(c \times a)$.

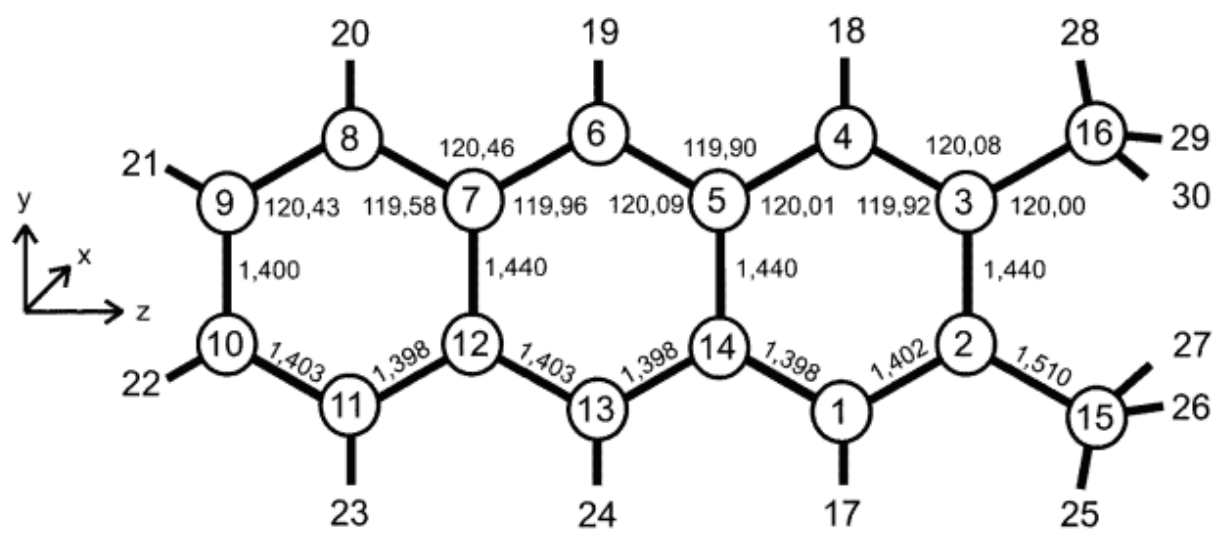

Figure 1. The atomic numbering scheme and the molecular geometry used for the rigid body refinement. The distances are given in $\AA$.

After it had been checked by temperature dependent Guinier powder measurements that no phase transition occurred between $93 \mathrm{~K}$ and $276 \mathrm{~K}$ (in contrast to 2,3dimethylnaphthalene), the unit cell parameters were also measured at $120 \mathrm{~K}$ (see table 2).

The program JIMPLAN [22] was used to obtain an electron density map in the molecular plane, figure 2. The crystal structure is displayed in stereoscopic projection in figure 3. (In the left part of the figure a statistical superposition of both molecular orientations is plotted to visualize dipolar disorder, whereas in the right part individual molecules with antiparallel orientation are displayed.)

Summarizing the $\mathrm{x}$-ray crystal structure results, the triclinic 2,3-DMA crystal with a bimolecular unit cell is dipolarly disordered so as to cancel a macroscopic dipole moment. Except for the dipolar disorder its crystal structure closely resembles that of the related tetracene molecule [23] which also is only slightly distorted from a monoclinic one (monoclinic structures are realized for the next smaller homologues, 2,3-dimethylnaphthalene [1] and anthracene [24], respectively). As might therefore be expected, 2,3-dimethylanthracene and tetracene [25], similar to the 2,3dimethylnaphthalene/anthracene pair [1] form mixed crystals over a wide range of concentrations. The extremely easy cleavage of 2,3DMA along (001) (as assigned by Laue photographs) is explainable on the basis of dipolar disorder: the molecular ends protruding from this surface can be either the methyl groups or the benzene ring $\mathrm{C}-\mathrm{H}$ groups (of the other end of the molecule). Since these orientations 
Table 1. 2,3-dimethylanthracene, fractional atomic coordinates of the two independent molecules A and B (marked by an asterisk) in the triclinic unit cell in one of the two possible orientations of the long molecular axis; dipolar disorder occurs by statistical inversion of the molecules at $[000]$ and $\left[\frac{1}{2} \frac{1}{2} 0\right]$. For the atomic numbering scheme see figure 1 .

\begin{tabular}{|c|c|c|c|c|c|c|c|c|c|}
\hline Atom & $\mathrm{X}$ & $\mathrm{Y}$ & $Z$ & U11 & U22 & U33 & U12 & U13 & U23 \\
\hline$C(01)$ & 0.0582 & -0.2340 & -0.1397 & 0.0494 & 0.0730 & 0.1361 & -0.0032 & 0.0259 & 0.0095 \\
\hline $\mathrm{C}(02)$ & 0.0003 & -0.1490 & -0.2423 & 0.0602 & 0.0860 & 0.1283 & -0.0063 & 0.0270 & 0.0049 \\
\hline $\mathrm{C}(03)$ & -0.0842 & 0.0620 & -0.2547 & 0.0550 & 0.0725 & 0.1627 & 0.0020 & 0.0339 & 0.0170 \\
\hline $\mathrm{C}(04)$ & -0.1088 & 0.1839 & -0.1636 & 0.0547 & 0.0736 & 0.1375 & -0.0119 & 0.0221 & 0.0095 \\
\hline $\mathrm{C}(05)$ & -0.0522 & 0.0994 & -0.0553 & 0.0422 & 0.0582 & 0.1291 & -0.0044 & 0.0137 & 0.0109 \\
\hline $\mathrm{C}(06)$ & -0.0752 & 0.2195 & 0.0392 & 0.0440 & 0.0656 & 0.1332 & 0.0017 & 0.0202 & 0.0167 \\
\hline $\mathrm{C}(07)$ & -0.0171 & 0.1344 & 0.1450 & 0.0469 & 0.0567 & 0.1362 & -0.0054 & 0.0183 & 0.0072 \\
\hline$C(08)$ & -0.0423 & 0.2546 & 0.2413 & 0.0592 & 0.0882 & 0.1616 & -0.0093 & 0.0393 & 0.0033 \\
\hline $\mathrm{C}(09)$ & 0.0156 & 0.1696 & 0.3439 & 0.0756 & 0.1005 & 0.1577 & -0.0020 & 0.0271 & 0.0128 \\
\hline$C(10)$ & 0.1000 & -0.0414 & 0.3564 & 0.0829 & 0.1225 & 0.1689 & -0.0139 & 0.0322 & 0.0209 \\
\hline$C(11)$ & 0.1247 & -0.1633 & 0.2653 & 0.0631 & 0.0776 & 0.1451 & -0.0048 & 0.0249 & 0.0218 \\
\hline$C(12)$ & 0.0681 & -0.0789 & 0.1569 & 0.0465 & 0.0666 & 0.1384 & -0.0076 & 0.0185 & 0.0141 \\
\hline$C(13)$ & 0.0910 & -0.1989 & 0.0624 & 0.0437 & 0.0545 & 0.1408 & -0.0019 & 0.0167 & 0.0173 \\
\hline$C(14)$ & 0.0329 & -0.1138 & -0.4330 & 0.0366 & 0.0596 & 0.1345 & -0.0067 & 0.0119 & 0.0117 \\
\hline$C(15)$ & 0.0270 & -0.2816 & -0.3414 & 0.0736 & 0.0909 & 0.1638 & 0.0097 & 0.0425 & -0.0055 \\
\hline$C(16)$ & -0.1435 & 0.1505 & -0.3683 & 0.0907 & 0.1203 & 0.1269 & 0.0105 & 0.0282 & 0.0348 \\
\hline $\mathrm{C}\left(01^{*}\right)$ & -0.3926 & -0.2697 & 0.1595 & 0.0489 & 0.0685 & 0.1184 & -0.0000 & 0.0194 & 0.0037 \\
\hline $\mathrm{C}\left(02^{*}\right)$ & -0.4187 & -0.3609 & 0.2519 & 0.0588 & 0.0814 & 0.1071 & -0.0000 & 0.0210 & 0.0062 \\
\hline $\mathrm{C}\left(03^{*}\right)$ & -0.5021 & -0.5746 & 0.2437 & 0.0503 & 0.0776 & 0.1235 & 0.0068 & 0.0257 & 0.0107 \\
\hline $\mathrm{C}\left(04^{*}\right)$ & -0.5580 & -0.6927 & 0.1426 & 0.0485 & 0.0681 & 0.1385 & 0.0056 & 0.0210 & 0.0039 \\
\hline $\mathrm{C}\left(05^{*}\right)$ & -0.5352 & -0.6018 & 0.0441 & 0.0438 & 0.0475 & 0.1195 & 0.0045 & 0.0221 & 0.0160 \\
\hline $\mathrm{C}\left(06^{*}\right)$ & -0.5906 & -0.7179 & -0.0603 & 0.0443 & 0.0642 & 0.1072 & 0.0050 & 0.0163 & 0.0047 \\
\hline $\mathrm{C}\left(07^{*}\right)$ & -0.5654 & -0.6264 & -0.1558 & 0.0472 & 0.0602 & 0.1241 & 0.0018 & 0.0227 & 0.0155 \\
\hline $\mathrm{C}\left(08^{*}\right)$ & -0.6235 & -0.7426 & -0.2624 & 0.0573 & 0.0747 & 0.1366 & 0.0030 & 0.0206 & -0.0008 \\
\hline $\mathrm{C}\left(09^{*}\right)$ & -0.5975 & -0.6513 & -0.3548 & 0.0815 & 0.1136 & 0.1381 & 0.0075 & 0.0299 & 0.0139 \\
\hline $\mathrm{C}\left(10^{*}\right)$ & -0.5141 & -0.4376 & -0.3465 & 0.0822 & 0.1130 & 0.1220 & 0.0096 & 0.0317 & 0.0172 \\
\hline $\mathrm{C}\left(11^{*}\right)$ & -0.4582 & -0.3195 & -0.2454 & 0.0614 & 0.0853 & 0.1189 & 0.0059 & 0.0234 & 0.0087 \\
\hline$C\left(12^{*}\right)$ & -0.4810 & -0.4105 & -0.1469 & 0.0452 & 0.0584 & 0.1068 & 0.0037 & 0.0167 & 0.0039 \\
\hline$C\left(13^{*}\right)$ & -0.4257 & -0.2943 & -0.0425 & 0.0457 & 0.0561 & 0.1165 & 0.0040 & 0.0188 & 0.0166 \\
\hline $\mathrm{C}\left(14^{*}\right)$ & -0.4508 & -0.3859 & 0.0530 & 0.0420 & 0.0601 & 0.1202 & 0.0029 & 0.0224 & 0.0077 \\
\hline$C\left(15^{*}\right)$ & -0.3579 & -0.2323 & 0.3620 & 0.0906 & 0.1092 & 0.1228 & -0.0129 & 0.0293 & -0.0156 \\
\hline$C\left(16^{*}\right)$ & -0.5260 & -0.6700 & 0.3470 & 0.0899 & 0.0974 & 0.1369 & -0.0044 & 0.0408 & 0.0257 \\
\hline
\end{tabular}

Table 2. Experimental lattice parameters for DMA- $\mathrm{h}_{14}$ from $\mathrm{x}$-ray data at $120 \mathrm{~K}$, and from neutron data at $10 \mathrm{~K}$ and calculated lattice parameters as well as lattice energies $\Delta H$ according to the models 1 and 2, respectively, as described in the text. ( $\Delta H$ is the calculated lattice energy at $0 \mathrm{~K}$.)

\begin{tabular}{llllllll}
\hline & $a(\mathrm{~nm})$ & $b(\mathrm{~nm})$ & $c(\mathrm{~nm})$ & $\alpha\left({ }^{\circ}\right)$ & $\beta\left({ }^{\circ}\right)$ & $\gamma\left({ }^{\circ}\right)$ & $\Delta H\left(\mathrm{kcal} \mathrm{mol}^{-1}\right)$ \\
\hline$T=120 \mathrm{~K}$ & $0.76835(7)$ & $0.59464(5)$ & $1.2443(47)$ & $96.32(11)$ & $104.62(5)$ & $89.62(1)$ & - \\
$T=10 \mathrm{~K}$ & $0.7638(25)$ & $0.5937(20)$ & $1.240(4)$ & $97.1(4)$ & $104.07(18)$ & $89.51(11)$ & - \\
Model 1 & 0.771 & 0.610 & 1.239 & 97.6 & 112.9 & 88.0 & -30.59 \\
Model 2 & 0.726 & 0.608 & 1.241 & 93.4 & 106.1 & 88.6 & -31.59 \\
\hline
\end{tabular}

follow in an irregular sequence, the (001) potential surfaces for van der Waals interactions are nonperiodic, thus excluding an optimal mutual approach. An interesting question which remained unsolved so far is that of the disorder correlations: what is the probability of a neighbouring molecule to point in the same direction as the considered one? Moreover, the question of how the phonons behave under the constraint of dipolar disorder is of fundamental interest. This topic will be studied in the next section. 


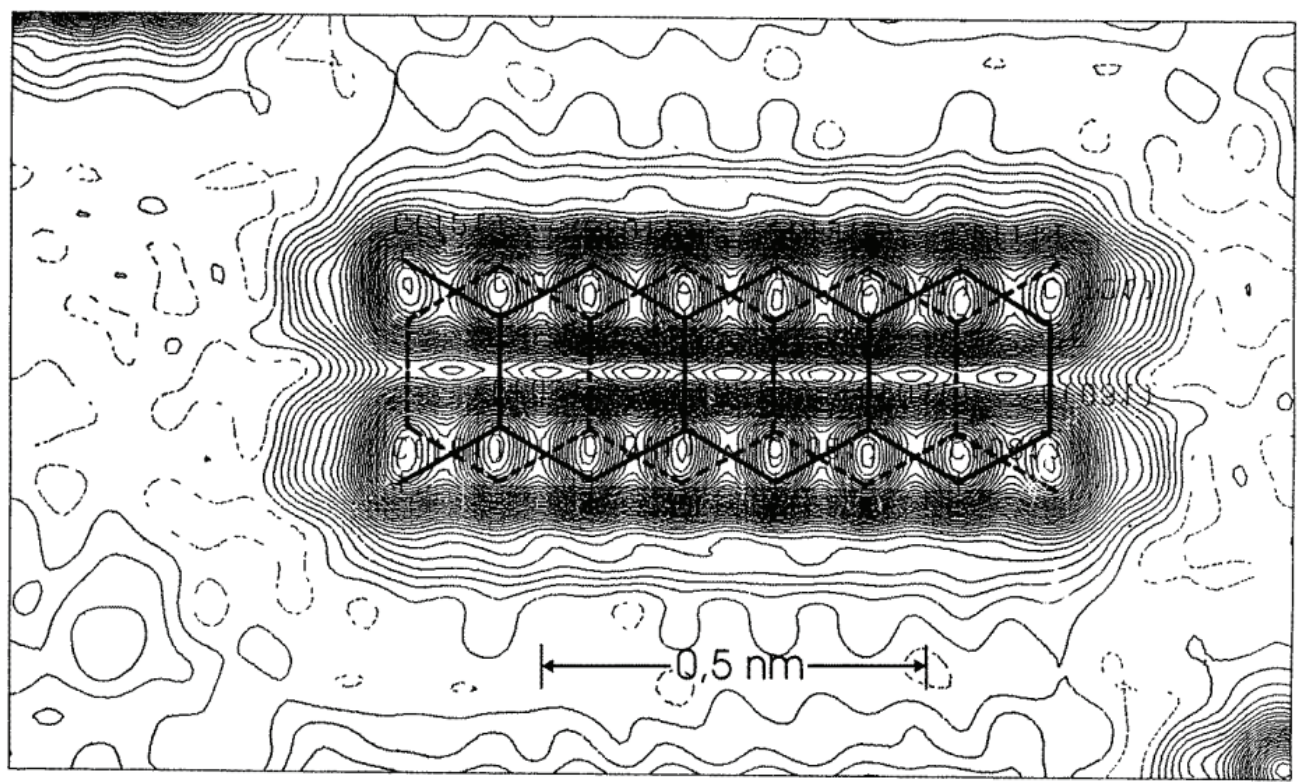

Figure 2. Electron density in the molecular plane of the two statistically superimposed orientations of the 2,3-dimethylanthracene molecule in the triclinic crystal structure. Contour lines are spaced 0.1 e $\AA^{-3}$.
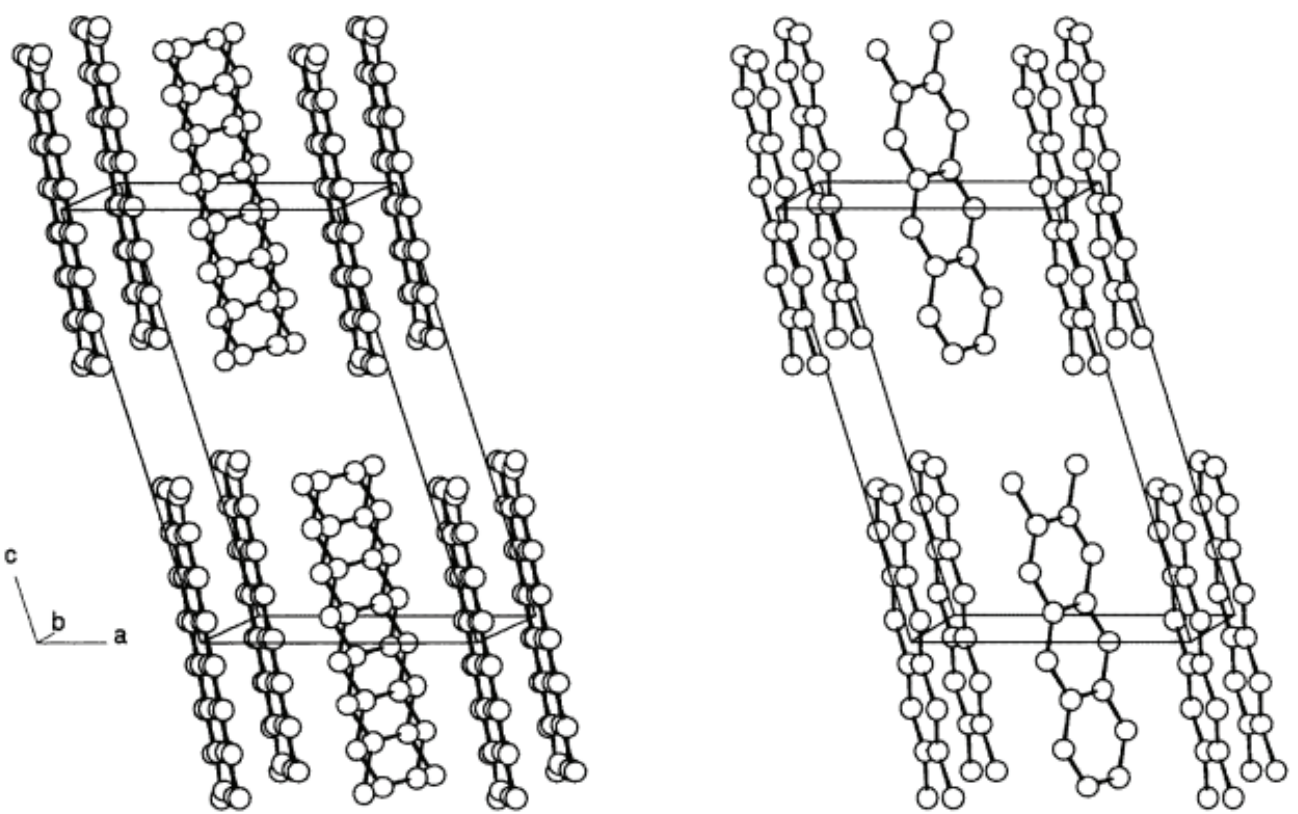

Figure 3. The crystal structure of 2,3-dimethylanthracene in stereoscopic projection (in the left part of the figure a statistical superposition of both molecular orientations is plotted to visualize dipolar disorder, whereas in the right part individual molecules with antiparallel orientation are displayed). 
The table of the observed and of the calculated structure factors is available upon request from one of the authors (NK).

\subsection{Incoherent inelastic neutron scattering (IINS)}

The IINS spectra of the powdered compounds $\mathrm{C}_{14} \mathrm{H}_{8}\left(\mathrm{CH}_{3}\right)_{2}$ ("2,3-DMA- $\mathrm{h}_{14}$ "), $\mathrm{C}_{14} \mathrm{D}_{8}\left(\mathrm{CH}_{3}\right)_{2}$ ("2,3-DMA-d,") and $\mathrm{C}_{14} \mathrm{D}_{8}\left(\mathrm{CD}_{3}\right)_{2}$ ("2,3-DMA-d $\mathrm{d}_{14}$ ") were measured at the inverted geometry time-of-flight spectrometer NERA-PR at the fast pulsed reactor IBR2 of the Joint Institute for Nuclear Research in Dubna [26]. The incident neutron energies were determined by measuring the neutron time of flight along the reactor-to-detector distance of $110.065 \mathrm{~m}$.

The rectangular sample holder of dimensions $15 \times 12 \mathrm{~cm}^{2}$ was placed in a cryostat cooled by liquid helium. The sample thickness was about $2 \mathrm{~mm}$ typically chosen for a transmission of the incident neutrons of around $90 \%$. In this way contributions from multiple scattering events become improbable. Typical measurement times ranged from 6 to $45 \mathrm{~h}$.

The time-of-flight spectra recorded at ten scattering angles between $25^{\circ}$ and $155^{\circ}$ were summed up to improve the statistics. For the monochromatization of the scattered neutrons an energy analysing system consisting of nitrogen-cooled beryllium filters and single crystal analysers of pyrolytic graphite was used. Together with the long flight path this resulted in a typical energy resolution between 2.5 and about $5.5 \%$ at an energy transfer between 25 $\mathrm{meV}$ and $500 \mathrm{meV}$, respectively.

The measured time of flight spectra were corrected for the background given by the empty cryostat, divided by the incident neutron spectrum, normalized to a measurement time of $10 \mathrm{~h}$ and to $0.1 \mathrm{~mol}$ of 2,3-DMA molecules. These normalized spectra were transformed to the amplitude-weighted phonon density of states $\bar{g}(\omega)$ by using the onephonon scattering formula of [27]. The transformed spectra of the differently deuterated samples are shown in figure 4 together with calculated spectra in the frequency range up to $10 \mathrm{THz}$.

A remarkable point is the missing energy gap in the frequency range between 3.5 and 5.0 THz. This behaviour was found for pure anthracene, too, but for pure naphthalene indeed a gap was observed. The missing of this gap means that in the low energy region the external as well as some low frequency internal phonons should be assumed to have similar energies. These phonon branches are then mixed together and show a substantial dispersion.

The differences in the general structure of the amplitude-weighted phonon densities of states $\bar{g}(\omega)$ in the energy regions below and above $5 \mathrm{THz}$ are related to the difference of the phonon dispersion in the crystal: in the low structured region below $5 \mathrm{THz}$ there exists a strong dependence of the phonon energies on the wave-vector $q$ (see figure 7 below). Above this frequency the dispersions of the phonon modes are small. Sometimes the bandwidths seen in this region are solely governed by the instrumental resolution of the spectrometer.

The different degrees of deuteration lead to different intensities of some phonon peaks of the 2,3-DMA the cause of which is related to the large incoherent scattering length of the hydrogen atoms. With the predominantly incoherent scattering $2,3-D M A-\mathrm{h}_{14}$ internal as well as external modes appear both with high intensities. For 2,3-DMA-d 8 one expects to see those modes with high intensity, which are related to the dynamics of the $\mathrm{CH}_{3}$ groups. Apart from external modes, these are especially those internal modes which represent librations of the $\mathrm{CH}_{3}$ groups or bending vibrations between the $\mathrm{CH}_{3}$ groups and the remainder of the molecule. 2,3-DMA- $\mathrm{d}_{14}$ scatters predominantly coherently. Yet in our ex- 


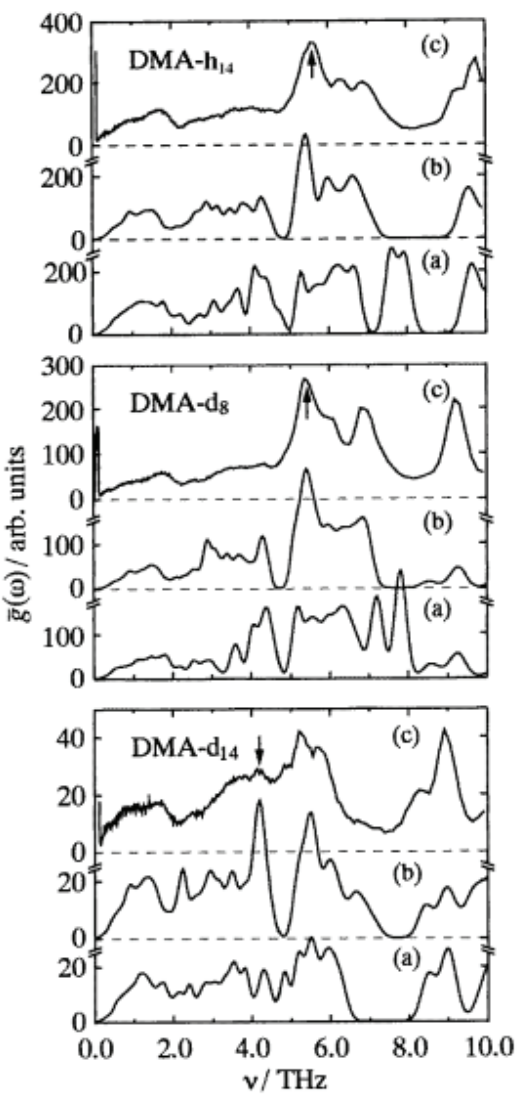

Figure 4. Measured and calculated amplitude weighted phonon density of states $\bar{g}(\omega)$ for 2,3-DMA- $\mathrm{h}_{14}, \mathrm{~d}_{8}, \mathrm{~d}_{14}$; (a) and (b) calculated $\bar{g}(\omega)$ after model 1 and model 2 respectively; (c) measured. The arrows indicate frequencies related to the librations of the methyl $\bar{g}(\omega)$. groups.

perimental situation, where the momentum transfer of the neutrons is quite high and the extension of the Brillouin zone is small, the so-called incoherent approximation is well established. In accordance with this approximation the reciprocal space is quite uniformly sampled for energy transfers larger than $1.5 \mathrm{THz}$ [28]. Compared to DMA- $\mathrm{h}_{14}$ the magnitude of the scattered intensity is reduced substantially in the case of DMA-d $\mathrm{d}_{14}$, because instead of the large incoherent scattering length of hydrogen now the small coherent scattering lengths of deuterium and carbon are effective. Furthermore frequency shifts appear which are due to isotopic effects. For external translational modes the expected frequency shifts compared with the corresponding frequencies of the undeuterated sample are of the order of $-1.9 \%$ for DMA- $\mathrm{d}_{8}$ and of $-3.2 \%$ for DMA-d 14 . Assuming a rigid molecule an averaged shift of -2.9 and $-6.0 \%$ can be expected for the librational modes of DMA- $\mathrm{d}_{8}$ and DMA- $\mathrm{d}_{14}$ respectively. By deuteration the_frequencies of the librations of the $\mathrm{CH} 3$ groups are reduced by a factor of the order of $1 / \sqrt{2}$, which is by far the largest frequency shift observed among all phonons. For convenience of the reader the frequencies related to the librations of the methyl groups are indicated by arrows in figure 4 .

Both the analysis of altered intensities and shifted frequencies in the spectra of the different deuterated samples help to identify the character of some internal modes. An assignment of the maxima in $\bar{g}(\omega)$ to internal modes of the free molecule is given in section 4 . 


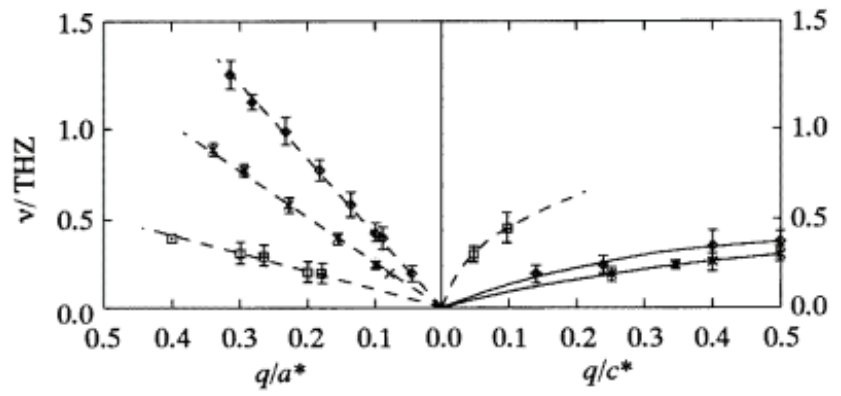

Figure 5. Measured phonon dispersion curves in directions $\mathrm{a}^{*}$ and $\mathrm{c}^{*}$. The lines are guides for the eyes. The uppermost curves are due to longitudinally polarized acoustic phonons in $E_{a^{*}}$ and $E_{c^{*}}$ directions, respectively, the middle curves represent transversally polarized phonons in the $E_{b^{*}}$ direction, and the lower curves are due to transversally polarized acoustic phonons in the $E_{c^{*}}$ direction (for the phonons in the $a^{*}$ direction) and in the $E_{a^{*}}$ direction (for the phonons in $c^{*}$ direction), respectively. The given statements for the directions of the polarization vectors are only approximately correct for phonons with wave vectors $q \rightarrow 0$, as described in the text. The given error bars indicate the measured intrinsic width (full width at half maximum) of the phonons. The error related to the phonon energy itself is smaller as the size of the different symbols.

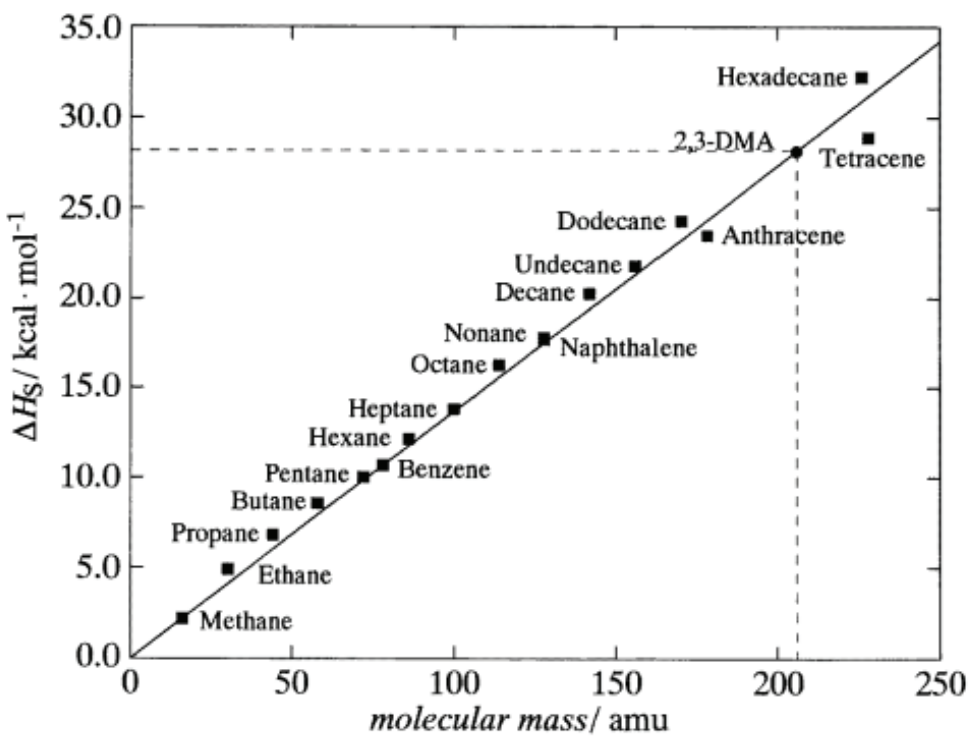

Figure 6. Energies of sublimations of $\Delta H_{s}$ some aliphatic and aromatic crystals as function of the molecular mass [34]. The expected value of 2,3-DMA is indicated by the dashed lines.

\subsection{Coherent inelastic neutron scattering}

Coherent inelastic neutron scattering experiments on a single crystal of 2,3-DMA-d14 were performed on the three-axis spectrometer V2 at the research reactor BER-2 of the Hahn- Meitner-Institut in Berlin [29] and on the instrument IN12 at the high flux reactor of the Institut Laue-Langevin in Grenoble [30]. Both spectrometers are installed at cold 

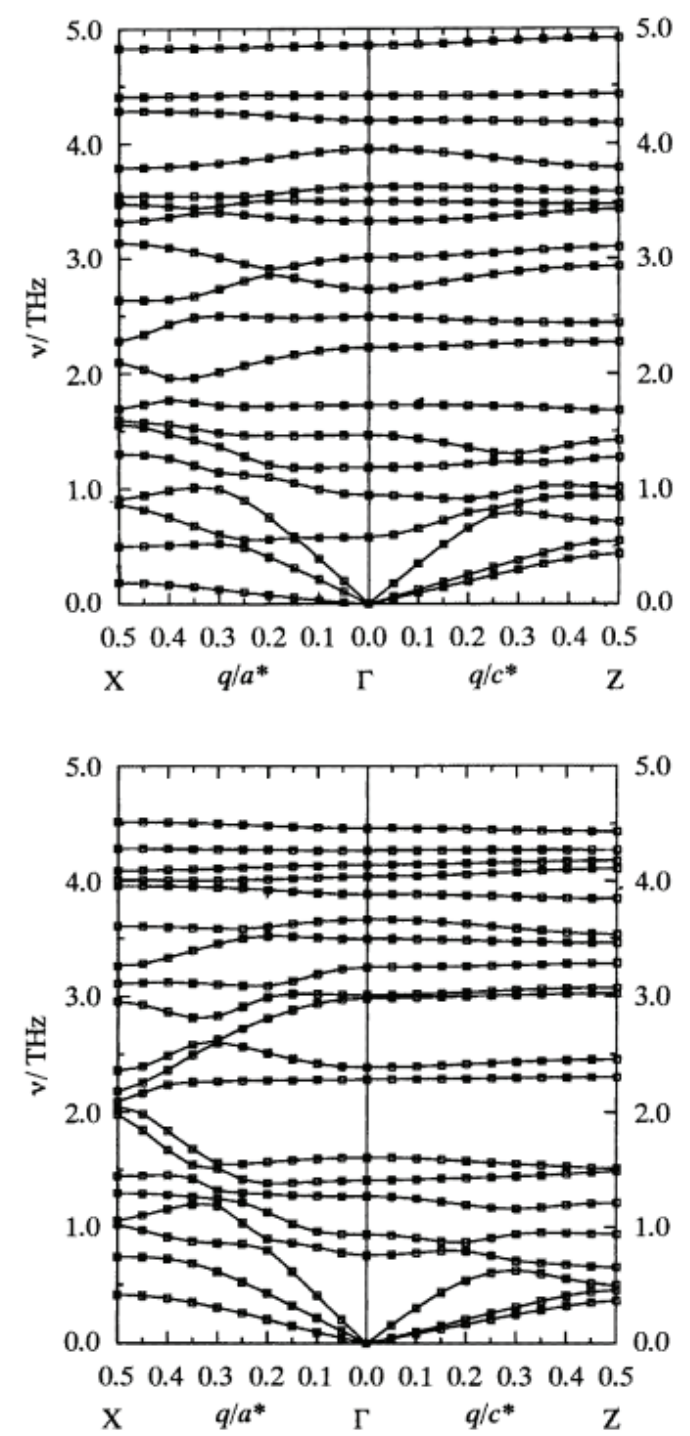

Figure 7. Calculated phonon dispersion curves for 2,3-DMA-d14 in $a^{*}$ and $\mathrm{c}^{*}$ directions. The upper curves were calculated for a unit cell with an up-up configuration (model 1), the lower ones for an up-down configuration (model 2).

neutron guides providing incident neutron energies in the range between 0.5 and $6.5 \mathrm{THz}$. The spectrometers are equipped with a $\mathrm{PG}(002)$ monochromator with variable vertical curvature and a $\mathrm{PG}(002)$ analyser. The mosaic spread of both monochromator and analyser is $0.4^{\circ}$. The horizontal divergence of the neutron beam was reduced by gadolinium coated Soller collimators behind the monochromator as well as before and behind the analyser. The divergence of all collimators was $1.0^{\circ}$. Most of the phonons were measured in the constant-energy mode with the wave number of the scattered neutrons kept fixed at 15 
$\mathrm{nm}^{-1}$ corresponding to a frequency of $1.13 \mathrm{THz}$. For suppression of higher order contaminations a nitrogen-cooled beryllium filter was placed in front of the analyser. This filter can be passed only by neutrons with energies lower than $1.26 \mathrm{THz}$.

It was expected that a single crystal of 2,3-DMA would show a great anisotropy of the thermal expansion coefficients like naphthalene or anthracene. Therefore, to avoid stresses and strains coming from temperature gradients, the crystal was cooled down and warmed up slowly at a rate of $10 \mathrm{~K} \mathrm{~h}^{-1}$ in the temperature range between 300 and $200 \mathrm{~K}$ and then at a rate of $15 \mathrm{~K} \mathrm{~h}^{-1}$ between 200 and $100 \mathrm{~K}$. At this temperature chosen for our experiments anharmonic effects and multi-phonon processes are sufficiently suppressed.

Because of the unfavourable macroscopic shape of the crystal the $a^{*}-c^{*}$ plane was chosen as the scattering plane. Furthermore from an interpretation of measurements of the diffuse x-ray scattering intensity we expected that phonons with high intensities can be found in this plane. Measurements of phonon dispersion curves were performed along the $a^{*}$ and $c^{*}$ directions.

The data recorded by the triple axis spectrometers were fitted by Gaussians representing the convolution of the resolution function of the instrument with the phonon line shape. Under the chosen experimental conditions anharmonic effects and thus line broadening according to a Lorentzian caused by finite phonon lifetime was assumed to be negligible in comparison to the finite phonon line width which follows from the dipolar disorder of the lattice. For the intrinsic phonon lineshapes related to disorder no simple general formula is available. Therefore as an approximation we assumed a Gaussian lineshape, too. The background was taken into account as a linear function of the wave vector $q$ or the frequency $v$, respectively. (The width of measured phonons differed for most cases not too much from the width due to the resolution of the instrument itself. A typical instrumental resolution was $0.035(0.08)$ in reduced units $\mathrm{q} / \mathrm{a}^{*}\left(q / c^{*}\right)$ in the $a^{*}\left(c^{*}\right)$ direction. Furthermore, background was a severe problem, because the phonon intensity was low. In reality there was no chance to decide whether the measured phonon line is better described by a Gaussian or a convolution of a Gaussian with a Lorentzian.) The results of measured phonon dispersion curves in the directions $a^{*}$ and $c^{*}$ are shown in figure 5. The intrinsic widths $\Delta E$ of the phonons were extracted from the measured widths and the calculated resolution functions of the three-axis spectrometers. $\Delta E$ is shown in figure 5 as a vertical "error bar" and is defined as the full width at half maximum. For phonons measured in the constant-energy mode these "error bars" in the vertical direction are obtained by taking into account the experimentally found dispersion of the phonon branch under consideration. A systematic increase of the linewidth with increasing phonon energy could not be observed. Yet phonon excitations for higher energies were lost in the background. This is the reason for the limited $v$-range of the observed dispersion curves presented in figure 5. The assignment of the predominant character of the motions of the molecules of the three measured acoustic phonon branches as indicated in the captions of figure 5 is due to the guide we obtained from the lattice dynamical calculations, presented in the next chapter, and due to intensities of phonons, measured under different conditions in different Brillouin zones.

\section{Lattice dynamical calculations}

Lattice dynamical calculations in the harmonic approximation are presented for two translationally invariant model crystals, for which we can easily calculate the phonon dispersion curves. In model 1 the methyl groups of the two molecules in the unit cell point in the same direction ("up-up" configuration) while in model 2 an opposite orientation was assumed ("up-down" configuration). Because the rigid molecule approximation is not longer 
valid in the case of 2,3-DMA, internal modes up to frequencies of $10 \mathrm{THz}$ were taken into account. The total potential energy of the crystal of $M$ molecules is given as

$$
V=\frac{1}{2} \sum_{m=1}^{M}\left(\sum_{p} f_{p} \varphi_{m p}^{2}+\sum_{n \neq m} V_{m n}\right)
$$

where $m$ and $n$ number the $M$ molecules in the crystal. The first sum in the parentheses describes the internal potential energy of the molecule $m$ as a superposition of the potential energies of all possible internal vibrations. These are expressed by $p$ internal coordinates $\varphi_{m p}$, which represent changes of interatomic distances, bonding angles, torsional angles etc, and related valence force constants $f_{p}$. The valence force constants were derived from gas data of smaller aromatic molecules [31, 32]. For the actual calculation of the phonon frequencies the force constants were considered as adjustable parameters allowing for the fact that the molecules might change their internal configuration in the potential field of the crystal. The second term refers to the interaction of the molecule $m$ with all other molecules $n$ in the crystal. The intermolecular potential energy between two different molecules $m$ and $n$ is written as a sum over the interaction potential between the atoms of these molecules:

$$
V_{m n}=\left(\sum_{k k^{\prime}} A_{k k^{\prime}} \exp \left(-B_{k k^{\prime}} r_{k k^{\prime}}\right)-C_{k k^{\prime}} r_{k k^{\prime}}^{-6}\right)_{m n} .
$$

Here $r_{k k^{\prime}}$, is the distance between the interacting atoms $k$ and $k$ ' of molecules $m$ and $n . A_{k k^{\prime}}$, $B_{k k}$, and $C_{k k}$, are empirical parameters which depend only on the chemical nature of the interacting atoms. For the interaction of atomic pairs $\mathrm{C}-\mathrm{C}, \mathrm{C}-\mathrm{H}$ and $\mathrm{H}-\mathrm{H}$ we used the Williams-IVa set of parameters [33] which was obtained by an adjustment to data of different aromatic and aliphatic molecular crystals. In the course of our calculations, it turned out that for 2,3-DMA a better agreement between the theoretical results and the measurements could be achieved if the parameters $A$ and $C$ of Williams were multiplied by a factor of 0.75 . The vibrational motions of a semi-rigid molecule in the gas phase consisting of $N$ atoms can be described by a number of $N_{c}$ independent constraints (like fixed distances or fixed angles between the atoms of the molecules etc). The number of degrees of freedom $N_{f}$ of a molecule therefore is reduced from $3 N$ to $N_{f}=3 N-N_{c}$. A proper description of the molecular motion therefore needs $N_{f}$ generalized coordinates and these are implemented in the general lattice dynamics calculation [34-37]. Finally the overall interaction energy between two atoms in the lattice is given by an expression, which can be calculated by a superposition of intramolecular and intermolecular force constants. (More details of the lattice dynamical model calculations are available upon request from one of the authors (JK).) Before any calculation of the phonon dispersion curves were started we optimized the orientation of the molecules in space as well as the lattice parameters in a systematic way by looking for a minimum in the static lattice energy $\Delta H$. In table 2 we compare the calculated lattice parameters with those measured at $10 \mathrm{~K}$ with the NERA-PR-spectrometer and with the $\mathrm{x}$-ray data obtained for DMA- $\mathrm{h}_{14}$ at $120 \mathrm{~K}$.

For most of the lattice parameters the agreement between measured and calculated values is quite good. If we compare the calculated lattice energy $\Delta H$ with measured energies of sublimations $\Delta H_{s}$ of some aliphatic and aromatic crystals [38] we find a nearly linear dependence of $\Delta H_{s}$ versus the mass of the molecules (see figure 6). According to this diagram we can expect that 2,3-DMA should have a value of $\Delta H_{s}$ around $28 \mathrm{kcal} \mathrm{mol}^{-1}$. This value is not far away from the value of $\Delta H$ calculated by our models. An experimental value of $\Delta H_{s}$ of 2,3-DMA is not known to our knowledge. After minimizing the total lattice energy with respect to the lattice parameters and the orientation of the molecules we calculated the amplitude weighted phonon density of states $\bar{g}(\omega)$ by sampling calcu- 
lated phonon energies and intensities at about 1800 different phonon wave vectors in the Brillouin zone. The results are shown in figure 4 together with the measured phonon densities of states. As can be seen, a much better agreement between theory and measurement is reached with model 2 . The reason for this is probably related with the strong interaction between the $\mathrm{H}$ atoms of the methyl groups of neighbouring molecules in model 1. This results also in a higher value of the lattice energy $\Delta H$ for model 1 after minimization. Figure 7 shows the corresponding calculated dispersion curves in $a^{*}$ and $\mathrm{c}^{*}$ directions for 2,3-DMA-d14. As far as we were able to measure the dispersion of the acoustic modes in the $a^{*}$ and $\mathrm{c}^{*}$ directions we can find a good agreement with the calculations of model 2, whereas model 1 is less favourable.

For the acoustic phonon branches we found for example that the polarization vectors for the $a^{*}$ phonons near the $\Gamma$-point $(q \rightarrow 0)$ are basically nearly parallel to the vectors of the reciprocal lattice. (Notice that the angles $\alpha^{*}, \beta^{*}$ and $\gamma^{*}$ of the unit cell of the reciprocal lattice are not very different from $90^{\circ}$ and that for the acoustic modes with $q \rightarrow 0$ the polarization vectors of the atoms of the three different modes have to be orthogonal.) This is different for phonons where $q \rightarrow 0$ is parallel to $c^{*}$. Here the transversally polarized modes deviate notable from the $a^{*}$ and $b^{*}$ directions.

From the differences of the frequencies of the acoustic modes calculated with model 1 and model 2, respectively, a crude estimate for the phonon line width for the dipolarly disordered crystal was deduced. (Notice that the lattice dynamics calculations are performed for translationally invariant ideal crystals.) We assume that the difference of the mode frequencies calculated for these two models can give representative information about the order of magnitude of the broadening of the phonon in the real, dipolarly disordered crystal. These calculated differences are (with one exception) in a range between 15 and $20 \%$ for the $c^{*}$ and $a^{*}$ directions of the acoustic branches and are in reasonably good agreement with the experimental data. The exception is connected with the lowest acoustic branch in the $a^{*}$ direction, where the calculated differences are around $75 \%$ and are definitely not in accordance with experimental data.

In figure 8 the displacement patterns of the internal modes and the related frequencies of a free 2,3-DMA- $\mathrm{h}_{14}$ molecule are presented as derived by our model calculations. Notice that the librations of the $\mathrm{CH}_{3}$ groups have zero frequencies in these calculations for reasons described in the next chapter. The change in frequencies due to deuteration is given in table 3. One has to be aware of the fact that by means of the interaction of the internal modes with the motion of neighbouring molecules via the term $V_{m n}$ of equations (1) and (2) the relevant displacement patterns as well as the frequencies change in the crystal. As an example we show in figure 9 a phonon mode at the $\Gamma$-point $(q=0)$ at $4.64 \mathrm{THz}$, which was calculated according to model 2 and which is closest in character to the $\mathrm{A}_{2}$ mode at $2.47 \mathrm{THz}$ of the free molecule. This example shows the influence of the coupling between modes of internal and external character.

\section{Discussion}

The lattice dynamical behaviour of 2,3-DMA can be satisfactorily reproduced by model calculations. Nevertheless it must be realized that the used Williams-IVa set of parameters cannot describe the motions of a flexible molecule in a fully correct way. This statement is based on the fact that in the presented model calculations the high librational frequencies of the methyl groups originate exclusively from the strong interaction of the $\mathrm{H}$ atoms of these groups with atoms of neighbouring molecules. The related intramolecular force constant $f_{k k^{\prime} k^{\prime \prime} k^{\prime \prime}}^{\text {torsion }}$ with $k=4, k^{\prime}=3, k^{\prime \prime}=16$ and $k^{\prime \prime \prime}=28$ was set to zero (see table 4 , figure 


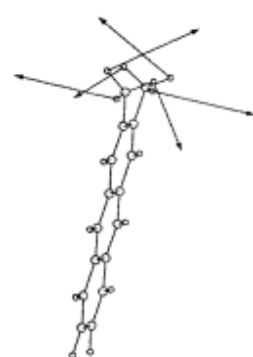

$\mathrm{A}_{2}$ $\mathrm{v}=0 \mathrm{THz}$

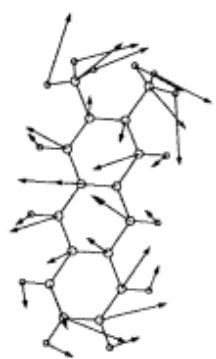

$\mathrm{B}_{2}$ $v=5.25 \mathrm{THz}$

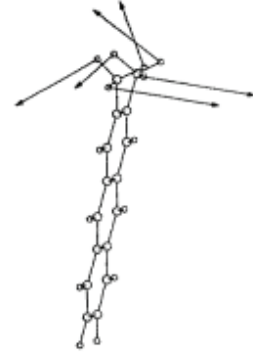

$\mathrm{B}_{1}$ $\mathrm{v}=0 \mathrm{THz}$

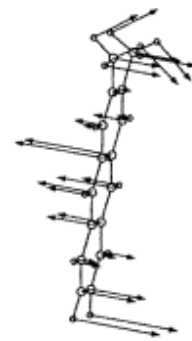

$\mathrm{B}_{1}$

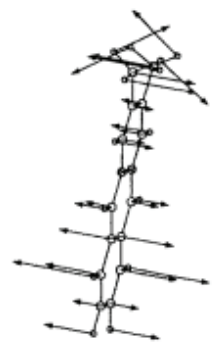

$\mathrm{A}_{2}$ $\mathbf{v}=2.47 \mathrm{THz}$

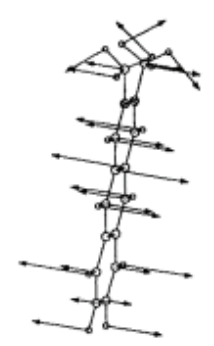

$\mathrm{A}_{2}$ $v=5.26 \mathrm{THz}$

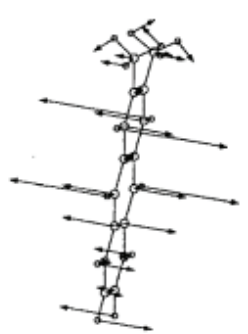

$\mathrm{A}_{2}$

$\mathrm{v}=8.88 \mathrm{THz}$

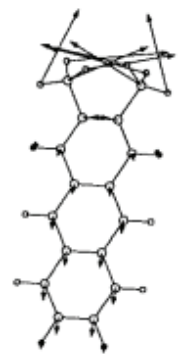

$A_{1}$

$v=5.68 \mathrm{THz}$

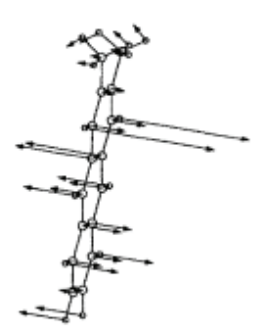

$\mathrm{B}_{1}$

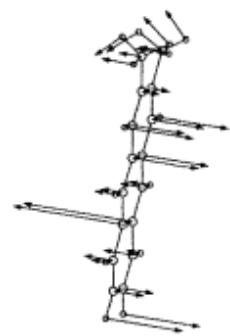

$\mathrm{B}_{\mathrm{t}}$

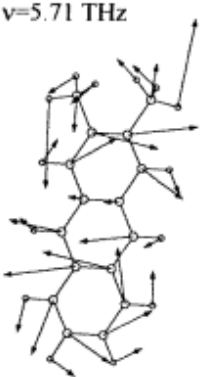

$\mathrm{B}_{2}$

$v=9.52 \mathrm{THz}$

Figure 8. Internal modes and frequencies of a free 2,3-DMA- $\mathrm{h}_{14}$-molecule in the frequency range up to $10 \mathrm{THz}$ as derived by the model calculations. For each mode and atom the direction and magnitude of the amplitudes are indicated by arrows.

1 and figure 11, where a visualization is given of how different atoms are involved in the definition of the force constants for a molecule in the gas phase). An internal rotation hindering potential as known from $o$-xylene [39] and 2,3-dimethylnaphthalene [40] in the gas phase could not be taken into account, because otherwise the frequencies of the librations of the methyl groups in the crystal would have been much too high, probably due to the strong increase of the chosen atom-atom potentials for distances lower than $0.2 \mathrm{~nm}$.

It is interesting to explore how the internal modes influence the lattice dynamics. In figure 10(a) we present calculated frequencies as well as intensities at the $\Gamma$-point $(q=0)$, for a crystal with rigid molecules. Notice that the three acoustic modes at frequency $v=0$ $\mathrm{THz}$ are not displayed. The displacement vectors for the different modes are characterized by a mixing between librations and translations. For example, only the mode with the highest energy can be simply described by a nearly pure libration around the molecular $z$-axis. 


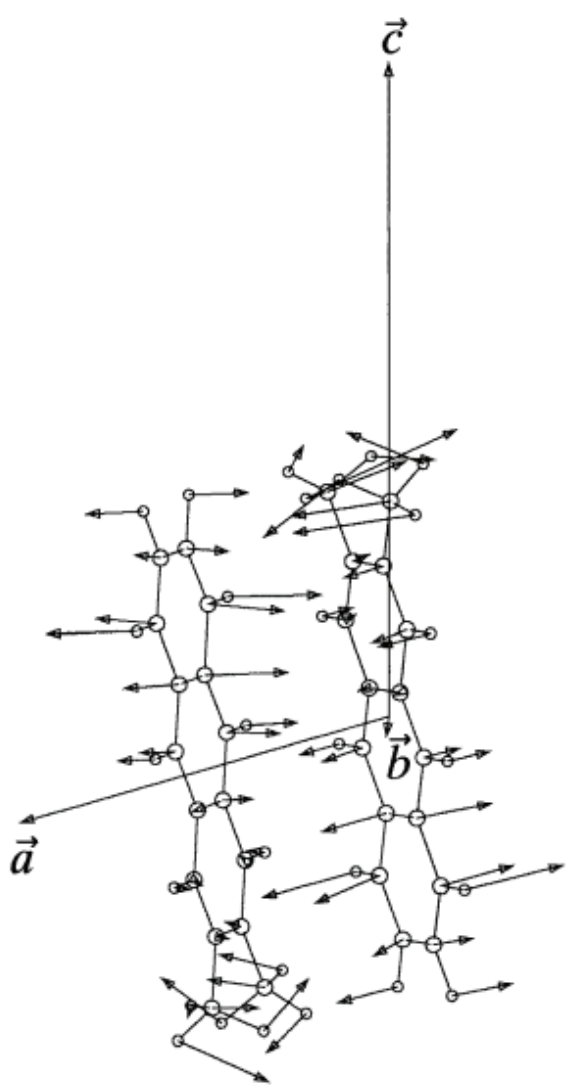

Figure 9. The displacement pattern of the phonon of $4.64 \mathrm{THz}$ at the $\Gamma$-point $(q=\mathbf{0})$, which is different in shape from the pattern of the free molecule at $2.47 \mathrm{THz}$ (see figure 8). Notice that the molecules are viewed along the $b$-axes of the unit cell, as indicated.

Table 3. Calculated mode frequencies for energies below $10 \mathrm{THz}$ for different degrees of deuteration of the free 2,3-DMA molecule. See figure 8 for the displacement patterns. A (B) modes are (anti-) symmetric under $\mathrm{C}_{2}$ rotation around the molecular $z$-axis (see figure 1); the index 1 (2) characterizes modes, which are (anti-) symmetric under reflection at the $x$ - $z$-plane of the molecule.

\begin{tabular}{llllllllllll}
\hline & \multicolumn{10}{c}{$v(\mathrm{THz})$} \\
\cline { 2 - 11 } Symmetry & $\mathrm{A}_{2}$ & $\mathrm{~B}_{1}$ & $\mathrm{~B}_{1}$ & $\mathrm{~A}_{2}$ & $\mathrm{~B}_{2}$ & $\mathrm{~A}_{2}$ & $\mathrm{~A}_{1}$ & $\mathrm{~B}_{1}$ & $\mathrm{~A}_{2}$ & $\mathrm{~B}_{1}$ & $\mathrm{~B}_{2}$ \\
\hline 2,3-DMA-h & 0 & 0 & 2.18 & 2.47 & 5.25 & 5.26 & 5.68 & 5.71 & 8.88 & 9.47 & 9.53 \\
2,3-DMA-d & 0 & 0 & 2.10 & 2.35 & 5.09 & 4.87 & 5.67 & 5.41 & 7.87 & 8.42 & 9.14 \\
$2,3-D M A-d_{14}$ & 0 & 0 & 2.04 & 2.23 & 4.79 & 4.72 & 4.99 & 5.32 & 7.79 & 8.37 & 8.83 \\
\hline
\end{tabular}

If methyl group librations are included in the model calculation, a substantial change occurs, as seen in figure 10(b). In this figure we have marked those modes where the displacement vectors resemble the $\mathrm{A}_{2}$ and $\mathrm{B}_{1}$ modes at frequencies $v=0 \mathrm{THz}$ of the free molecules by filled and open dots, respectively (see table 3, figures 8 and 10(c), where the fre- 
Table 4. Intramolecular force constants (values in units of $100 \mathrm{~N} \mathrm{~m}^{-1}$ ). For more details see the text and figure 11. The numbering of the atoms is given according to the scheme in figure 1. The results of the lattice dynamical calculations shown in figures 4 and 7 are performed with models, in which for $f>6$ units the corresponding distortion is assumed to be rigid.

\begin{tabular}{|c|c|c|c|c|c|c|c|c|c|c|c|}
\hline$k$ & $k^{\prime}$ & $f_{k k^{\prime}}^{\text {stetch }}$ & $k$ & $k^{\prime}$ & $f_{k k^{\prime}}^{\text {stretch }}$ & $k$ & $k^{\prime}$ & $f_{k k^{\prime}}^{\text {stretch }}$ & $k$ & $k^{\prime}$ & $f_{k k^{\prime}}^{s t r c h}$ \\
\hline 1 & 2 & 5.9 & 6 & 19 & 4.79 & 3 & 5 & 0.61 & 10 & 12 & 0.61 \\
\hline 2 & 3 & 4.2 & 8 & 20 & 4.79 & 4 & 14 & 0.61 & 11 & 13 & 0.44 \\
\hline 3 & 4 & 5.9 & 9 & 21 & 4.79 & 4 & 6 & 0.44 & 12 & 14 & 0.61 \\
\hline 4 & 5 & 5.9 & 10 & 22 & 4.79 & 5 & 7 & 0.61 & 17 & 2 & 0.32 \\
\hline 5 & 6 & 5.9 & 11 & 23 & 4.79 & 5 & 12 & 0.09 & 17 & 14 & 0.32 \\
\hline 6 & 7 & 5.9 & 13 & 24 & 4.79 & 5 & 13 & 0.51 & 18 & 3 & 0.32 \\
\hline 7 & 8 & 5.9 & 15 & 25 & 4.8 & 6 & 8 & 0.44 & 18 & 5 & 0.32 \\
\hline 8 & 9 & 5.9 & 15 & 26 & 4.8 & 6 & 14 & 0.61 & 19 & 5 & 0.32 \\
\hline 9 & 10 & 4.2 & 15 & 27 & 4.8 & 6 & 12 & 0.61 & 19 & 7 & 0.32 \\
\hline 10 & 11 & 5,9 & 16 & 28 & 4.8 & 6 & 13 & 0.09 & 20 & 7 & 0.32 \\
\hline 11 & 12 & 5.9 & 16 & 29 & 4.8 & 7 & 14 & 0.09 & 20 & 9 & 0.32 \\
\hline 12 & 13 & 5.9 & 16 & 30 & 4.8 & 7 & 13 & 0.51 & 21 & 8 & 0.32 \\
\hline 13 & 14 & 5.9 & 1 & 3 & 0.61 & 7 & 9 & 0.61 & 21 & 10 & 0.32 \\
\hline 14 & 1 & 5.9 & 1 & 4 & 0.09 & 7 & 10 & 0.09 & 22 & 9 & 0.32 \\
\hline 7 & 12 & 4.2 & 1 & 5 & 0.51 & 7 & 11 & 0.51 & 22 & 11 & 0.32 \\
\hline 5 & 14 & 4.2 & 1 & 13 & 0.44 & 8 & 10 & 0.61 & 23 & 10 & 0.32 \\
\hline 2 & 15 & 5.9 & 2 & 4 & 0.61 & 8 & 11 & 0.09 & 23 & 12 & 0.32 \\
\hline 3 & 16 & 5.9 & 2 & 5 & 0.09 & 8 & 12 & 0.61 & 24 & 12 & 0.32 \\
\hline 1 & 17 & 4.79 & 2 & 14 & 0.61 & 9 & 11 & 0.61 & 24 & 14 & 0.32 \\
\hline 4 & 18 & 4.79 & & 14 & 0.09 & 9 & 12 & 0.90 & & & \\
\hline$k$ & $k^{\prime}$ & $k^{\prime \prime}$ & $f_{k k^{\prime} k^{\prime \prime}}^{b e n d}$ & $k$ & $k^{\prime}$ & $k^{\prime \prime}$ & $f_{k k^{\prime} k^{\prime \prime}}^{\text {bend }}$ & $k$ & $k^{\prime}$ & $k^{\prime \prime}$ & $f_{k k^{\prime} k^{\prime \prime}}^{\text {bend }}$ \\
\hline 17 & 1 & 14 & 0.456 & 6 & 7 & 8 & 0.78 & 12 & 13 & 14 & 0.763 \\
\hline 17 & 1 & 2 & 0.446 & 6 & 7 & 12 & 0.78 & 12 & 13 & 24 & 0.456 \\
\hline 14 & 1 & 2 & 0.763 & 8 & 7 & 12 & 0.78 & 14 & 13 & 24 & 0.456 \\
\hline 1 & 2 & 3 & 0.763 & 7 & 8 & 9 & 0.763 & 13 & 14 & 1 & 0.78 \\
\hline 1 & 2 & 15 & 0.463 & 7 & 8 & 20 & 0.456 & 13 & 14 & 5 & 0.78 \\
\hline 3 & 2 & 15 & 0.40 & 9 & 8 & 20 & 0.446 & 1 & 14 & 5 & 0.78 \\
\hline 2 & 3 & 4 & 0.763 & 8 & 9 & 10 & 0.763 & 2 & 15 & 25 & 10.346 \\
\hline 2 & 3 & 16 & 0.40 & 8 & 9 & 21 & 0.446 & 2 & 15 & 26 & 10.646 \\
\hline 4 & 3 & 16 & 0.463 & 10 & 9 & 21 & 0.446 & 2 & 15 & 27 & 10.646 \\
\hline 3 & 4 & 5 & 0.763 & 9 & 10 & 11 & 0.763 & 25 & 15 & 27 & 10.3 \\
\hline 3 & 4 & 18 & 0.446 & 9 & 10 & 22 & 0.446 & 26 & 15 & 27 & 10.3 \\
\hline 5 & 4 & 18 & 0.456 & 11 & 10 & 22 & 0.446 & 25 & 15 & 26 & 10.5 \\
\hline 4 & 5 & 6 & 0.78 & 10 & 11 & 12 & 0.763 & 3 & 16 & 28 & 10.346 \\
\hline 4 & 5 & 14 & 0.78 & 10 & 11 & 23 & 0.446 & 3 & 16 & 29 & 10.346 \\
\hline 6 & 5 & 14 & 0.78 & 12 & 11 & 23 & 0.456 & 3 & 16 & 30 & 10.346 \\
\hline 5 & 6 & 7 & 0.763 & 11 & 12 & 13 & 0.78 & 28 & 16 & 30 & 10.5 \\
\hline 5 & 6 & 19 & 0.456 & 11 & 12 & 7 & 0.78 & 29 & 16 & 30 & 10.5 \\
\hline 7 & 6 & 19 & 0.456 & 13 & 12 & 7 & 0.78 & 28 & 16 & 29 & 10.5 \\
\hline
\end{tabular}

quencies, mode-characters and related symbols of the internal modes are presented). The two lowest frequencies marked in this way are related to the $A_{2}$, the two others to the $B_{1}$ mode. For the $\mathrm{A}_{2}$ modes a large difference between the frequencies is observed. As a result the lower frequency of the $\mathrm{A}_{2}$ mode is related to a displacement pattern, where the rest of the molecule librates approximately around the $z$-axis in opposite direction as the $\mathrm{CH}_{3}$ groups do, whereas for the higher frequency both librations are in the same direction. 
Table 4. continued.

\begin{tabular}{|c|c|c|c|c|c|c|c|c|c|}
\hline$k$ & $k^{\prime}$ & $k^{\prime \prime}$ & $k^{\prime \prime \prime}$ & $f_{k k^{\prime} k^{\prime \prime} k^{\prime \prime \prime}}^{\text {torsion }}$ & $k$ & $k^{\prime}$ & $k^{\prime \prime}$ & $k^{\prime \prime \prime}$ & $f_{k k^{\prime} k^{\prime \prime} k^{\prime \prime \prime}}^{\text {torsion }}$ \\
\hline 1 & 2 & 3 & 4 & 0.12 & 12 & 7 & 8 & 9 & 0.12 \\
\hline 2 & 3 & 4 & 5 & 0.12 & 1 & 2 & 15 & 25 & 0.00 \\
\hline 3 & 4 & 5 & 14 & 0.12 & 4 & 3 & 16 & 28 & 0.00 \\
\hline 4 & 5 & 14 & 1 & 0.07 & 15 & 2 & 3 & 16 & 0.12 \\
\hline 5 & 14 & 1 & 2 & 0.12 & 16 & 3 & 4 & 18 & 0.12 \\
\hline 14 & l & 2 & 3 & 0.12 & 18 & 4 & 5 & 6 & 0.12 \\
\hline 5 & 6 & 7 & 12 & 0.12 & 4 & 5 & 6 & 19 & 0.12 \\
\hline 6 & 7 & 12 & 13 & 0.07 & 19 & 6 & 7 & 8 & 0.12 \\
\hline 7 & 12 & 13 & 14 & 0.12 & 6 & 7 & 8 & 20 & 0.12 \\
\hline 12 & 13 & 14 & 5 & 0.12 & 20 & 8 & 9 & 21 & 0.12 \\
\hline 13 & 14 & 5 & 6 & 0.07 & 21 & 9 & 10 & 22 & 0.12 \\
\hline 14 & 5 & 6 & 7 & 0.12 & 22 & 10 & 11 & 23 & 0.12 \\
\hline 7 & 8 & 9 & 10 & 0.12 & 23 & 11 & 12 & 13 & 0.12 \\
\hline 8 & 9 & 10 & 11 & 0.12 & 11 & 12 & 13 & 24 & 0.12 \\
\hline 9 & 10 & 11 & 12 & 0.12 & 24 & 13 & 14 & 1 & 0.12 \\
\hline 10 & 11 & 12 & 7 & 0.12 & 13 & 14 & 1 & 17 & 0.12 \\
\hline 11 & 12 & 7 & 8 & 0.07 & 17 & 1 & 2 & 15 & 0.12 \\
\hline$k$ & $k^{\prime}$ & $k^{\prime \prime}$ & $k^{\prime \prime \prime}$ & $f_{k k^{\prime} k^{\prime \prime} k^{\prime \prime \prime}}^{\text {out of plane }}$ & $k$ & $k^{\prime}$ & $k^{\prime \prime}$ & $k^{\prime \prime \prime}$ & $f_{k k^{\prime} k^{\prime \prime} k^{\prime \prime \prime \prime}}^{\text {out planc }}$ \\
\hline 14 & 17 & 2 & 1 & 0.15 & 12 & 24 & 14 & 13 & 0.15 \\
\hline 1 & 3 & 15 & 2 & 0.15 & 5 & 13 & 1 & 14 & 0.2 \\
\hline 2 & 4 & 16 & 3 & 0.15 & 1 & 2 & 3 & 15 & 10.2 \\
\hline 3 & 18 & 5 & 4 & 0.15 & 2 & 3 & 4 & 16 & 10.2 \\
\hline 14 & 4 & 6 & 5 & 0.2 & 3 & 4 & 5 & 18 & 10.25 \\
\hline 5 & 19 & 7 & 6 & 0.15 & 5 & 6 & 7 & 19 & 10.25 \\
\hline 12 & 6 & 8 & 7 & 0.2 & 7 & 8 & 9 & 20 & 10.25 \\
\hline 7 & 20 & 9 & 8 & 0.15 & 8 & 9 & 10 & 21 & 10.25 \\
\hline 8 & 21 & 10 & 9 & 0.15 & 9 & 10 & 11 & 22 & 10.25 \\
\hline 11 & 22 & 9 & 10 & 0.15 & 10 & 11 & 12 & 23 & 10.25 \\
\hline 10 & 23 & 12 & 11 & 0.15 & 12 & 13 & 14 & 24 & 10.25 \\
\hline 11 & 13 & 7 & 12 & 0.2 & 14 & 1 & 2 & 17 & 10.25 \\
\hline
\end{tabular}

If we include now all internal modes with frequencies below $10 \mathrm{THz}$ in the gas phase we end up with figure 10(d). In this figure we indicated by the corresponding symbols those phonons for which the displacement patterns of the internal modes in the gaseous state give an important contribution to the actual displacement patterns in the solid state. Especially for the low frequency internal gas phase modes substantial energy shifts can be found. As mentioned, the calculations presented here are restricted to the $\Gamma$-point. Nevertheless the diagram of figure 10(d) can be compared with the measured amplitudeweighted phonon density of states $\bar{g}(\omega)$ for DMA-h ${ }_{14}$ (see figure 4 , DMA- $\mathrm{h}_{14}$ ) and gives a guide to in which region of the phonon energies the formerly internal vibrations of the gas phase contribute substantially to $\bar{g}(\omega)$.

The actual values and the mutual ordering of mode frequencies and their accompanying displacement patterns depend sensitively on the model parameters used for both the internal and external forces. The force constants used in our calculations are derived by slight variations of otherwise known parameters and give a consistent description of the amplitude-weighted phonon density of states and by a comparison of different idealized model crystals a crude estimate for measured phonon widths. The measured phonon width is a convolution of real phonon lifetime effects and of a width related to disorder. We were 

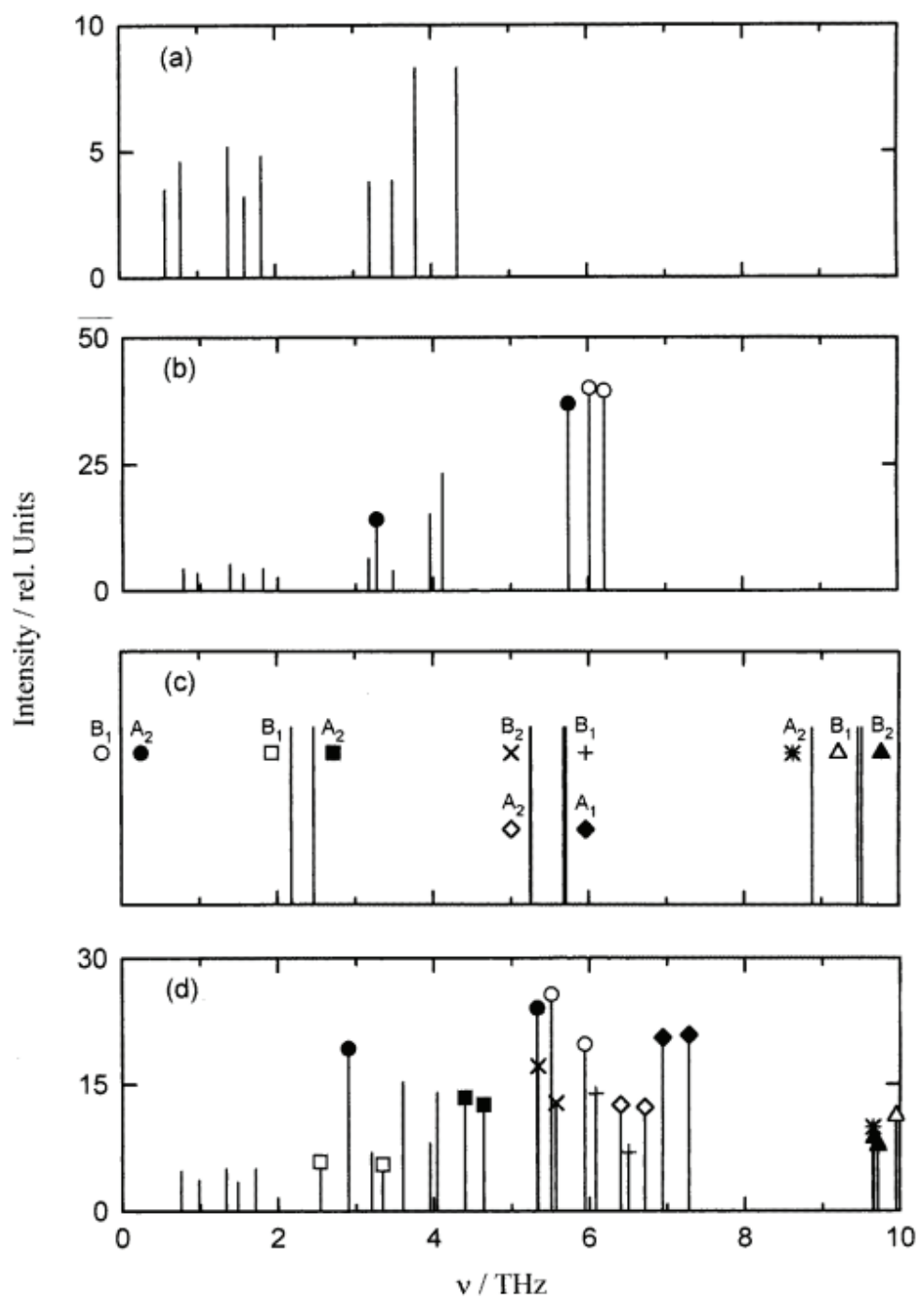

Figure 10. (a) Frequencies of lattice modes for a crystal with rigid molecules. (b) Frequencies of phonons for a crystal where the molecules are rigid with the exception of the methyl rotations. (c) Calculated frequencies of the internal modes in the gas phase for 2,3-DMA-h ${ }_{14}$ (see table 3). The symbols for the different modes are used in (b) and (d). For more details see the text. (d) Frequencies of phonons calculated for a crystal with flexible molecules. The internal modes of (c) are added. All frequencies of the phonon modes are calculated for model 2 and for 2,3-DMA- $\mathrm{h}_{14}$ at the $\Gamma$-point $(q=0)$ for (a), (b) and (d). Notice that the three acoustic phonon modes with zero frequencies are not shown in (a), (b) and (d). The heights of the bars in (a), (b) and (d) give the mean values of the calculated neutron cross-sections of the $\Gamma$-modes. The heights of the bars in (c) have no meaning; these bars are only guides to the eyes for vibrational frequencies.

not able to give a true separation for these two contributions. Neglecting the real phonon lifetime effect our examinations give an upper bond for the contribution due to disorder. Comparisons can be made with some measured phonon widths of pure anthracene 


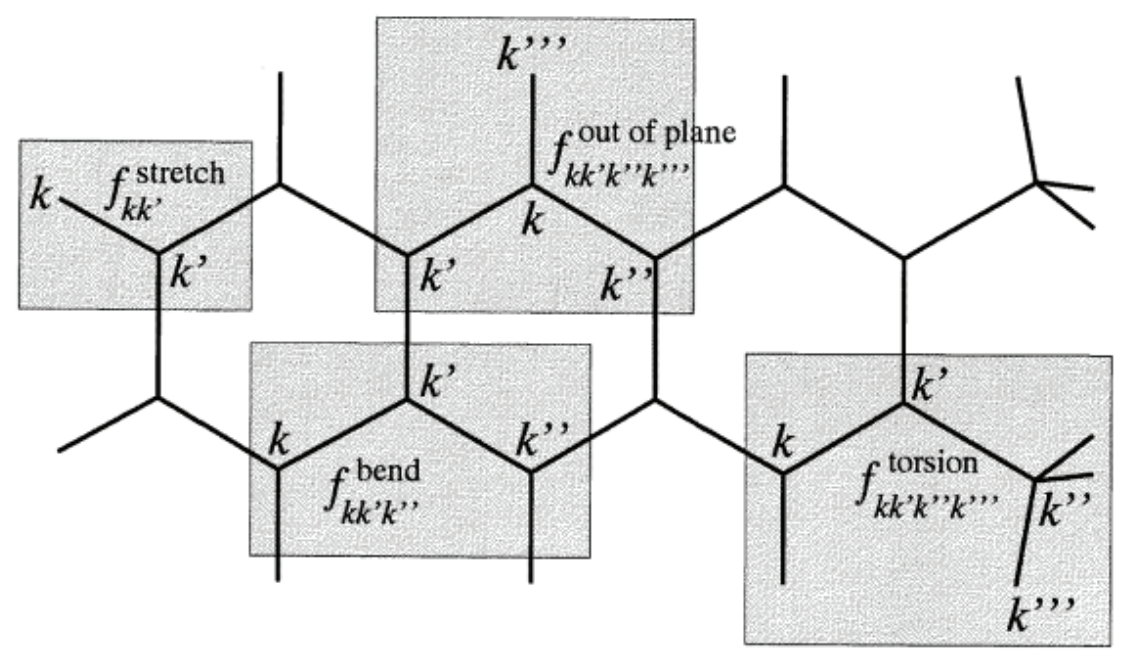

Figure 11. Visualization of the intramolecular force constants. $f_{k k^{\prime}}^{\text {stretch }}$ is the stretch force constant between atom $k$ and $k^{\prime}, f_{k k^{\prime} k^{\prime \prime}}^{b e n}$ is the force constant describing the bending of the row of the atoms $k, k^{\prime}$, and $k^{\prime \prime}$ in the plane of the molecule, $f_{k k^{\prime} k^{\prime \prime} k^{\prime \prime}}^{\text {torsion }}$ describes the torsion of the axis between atoms $k^{\prime}$ and $k^{\prime \prime}$ if the atoms $k$ and $k^{\prime \prime \prime}$ make out of plane displacements, and $f_{k k^{\prime} k^{\prime \prime} k^{\prime \prime \prime}}^{\text {out }}$ describes the change of the angle of bond $k-k^{\prime \prime \prime}$ perpendicular to the plane containing $k, k^{\prime}$, and $k^{\prime \prime \prime}$. The numbering of the atoms is indicated in figure 1 .

[41] at $100 \mathrm{~K}$. For different phonon branches with phonon energies below $\sim 1.6 \mathrm{THz}$ these measured widths scatter around $0.023-0.095 \mathrm{THz}$ (FWHM); but unfortunately no data are available for phonon branches related to our measurements in $\mathrm{a}^{*}$ and $\mathrm{c}^{*}$ directions.

\section{Acknowledgments}

This work was supported by the Bundesministerium für Forschung und Technologie (BMFT), grant No 03-KA3BAY-4.

\section{References}

[1] Karl N, Heym H and Stezowski J J 1985 Mol. Cryst. Liq. Cryst. 131163

[2] Schreier R to be published

[3] Dörr M, Kalus J, Monkenbusch M, Natkaniec I and Schmelzer U 1996 Physica B 219/220 368

[4] Dorner B, Bokhenkov E L, Chaplot S L, Kalus J, Natkaniec I, Pawley G S, Schmelzer U and Sheka E F 1982 J. Phys. C: Solid State Phys. 152353

[5] Allen C F H and Bell A 1955 Org. Synth. Coll. 3310

[6] Elbs K and Eurich H 1887 Ber. Deutsch. Chem. Ges. 201361

[7] Baker W, Banks R, Lyon D R and Mann F G 1945 J. Chem. Soc. 27

[8] Orchin M 1944 J. Am. Chem. Soc. 66535

[9] Barnett E B and Marrison F C 1931 Ber. Deutsch. Chem. Ges. 64535

[10] Kaupp G 1971 Chimia 25230

[11] de Diesbach H and von der Weid E 1927 Helv. Chim. Acta 10886 
[12] Ried W and Boennighausen K H 1961 Justus Liebigs Ann. Chem. 63961

[13] Vogel A 1956 A Text Book of Practical Organic Chemistry 3rd edn. (London: Longmans Green) p 739

[14] Fieser L F and Fieser M 1935 J. Am. Chem. Soc. 571679

[15] Snyder H R and Werber F X 1950 J. Am. Chem. Soc. 722965

[16] Fieser L F and Newton L W 1942 J. Am. Chem. Soc. 64917

[17] Kalus J, Gerlach H, Schleifer J, Woerlen F, Voss G, Godlewska M, Natkaniec I, Karl N and Prager M 1986 Phys. Status Solidi b 13453

[18] Weber J A and Boggs A D 1952 J. Chem. Educ. 29363 Fieser L F and Seligman A M 1934 J. Am. Chem. Soc. $\mathbf{5 6} 2690$

[19] Allen CFH and Bell A 1955 Org. Synth. Coll. 3312

[20] Karl N 1980 Crystals—Growth, Properties and Applications ed H C Freyhardt (Berlin: Springer) pp 1-100

[21] Stewart J M (ed) 1976 The X-ray system—version of 1976 Technical Report of the Computer Science Center, University of Maryland

[22] Hansen N K Program JIMPLAN A program for calculating Fourier summations in arbitrarily chosen planes, Buffalo 1976

[23] Robertson J M, Sinclair V C, Trotter J 1961 Acta Crystallogr. 14697 Campbell R B, Robertson J M and Trotter J 1962 Acta Crystallogr. 15289

[24] Mathieson A McL, Robertson J M and Sinclair V C 1950 Acta Crystallogr. 3245 Mason R 1964 Acta Crystallogr. 17547

[25] Karl N 1984 Materials Science vol 10, No 3 (Wroclaw: University of Wroclaw) pp 365-78

[26] Natkaniec I, Bragin S I, Brankowski J and Mayer J 1994 Proc. 12th Meeting Int. Collaboration on Advanced Neutron Sources vol 1, Rutherford Appleton Laboratory, Chilton

[27] Janik J A, Bajorek A, Janik J M, Natkaniec I, Parlinski K and Sudnik-Hrynkiewicz M 1968 Acta Phys. Pol. 33419

[28] Leadbetter A J 1973 Chemical Application of Thermal Neutron Scattering ed BRM Willis (Oxford: Oxford University Press) pp 146-72

[29] 1992 Instrumentation at the Research Reactor Berlin Hahn-Meitner-Institut, Berlin

[30] Ibel K (ed) 1994 The Yellow Book (Grenoble: ILL Grenoble)

[31] Scherer J R 1962 J. Chem. Phys. 363308

[32] Cyvin S J 1982 J. Mol. Struct. 79423

[33] Williams D E 1967 J. Chem. Phys. 474680

[34] Mills I M 1969 Chem. Phys. Lett. 3267

[35] Monkenbusch M 1987 Acta Crystallogr. A 43198

[36] Califano S, Schettino V and Neto N 1981 Lattice Dynamics of Molecular Crystals (Berlin: Springer)

[37] Neto N 1984 Chem. Phys. 9189

[38] Kitaigorodsky A I 1973 Molecular Crystals and Molecules (New York: Academic)

[39] Breen P J, Warren J A, Bernstein E R and Seeman J I 1987 J. Chem. Phys. 871917

[40] Tan X Q, Clouthier D J, Judge R H, Plusquellic D F, Torner J L and Pratt D W 1991 J. Chem. Phys. 957862

[41] Jordan JFJ, Kalus J, Schmelzer U and Eckold G 1989 Phys. Status Solidi b 15589 